\title{
Computing accurate forces in quantum Monte Carlo using Pulay's corrections and energy minimization
}

\author{
Mosé Casalegno ${ }^{\text {a) }}$ \\ Dipartimento di Chimica Fisica ed Elettrochimica, Universitá degli Studi di Milano, via Golgi 19, \\ 20133 Milano, Italy and Department of Chemistry and Laboratory for Research on the Structure of Matter, \\ University of Pennsylvania, Philadelphia, Pennsylvania 19104-6323 \\ Massimo Mella ${ }^{\text {b) }}$ \\ Dipartimento di Chimica Fisica ed Elettrochimica, Universitá degli Studi di Milano, via Golgi 19, \\ 20133 Milano, Italy
}

Andrew M. Rappe ${ }^{\mathrm{c})}$

Department of Chemistry and Laboratory for Research on the Structure of Matter, University of Pennsylvania, Philadelphia, Pennsylvania 19104-6323

(Received 19 November 2002; accepted 30 January 2003)

\begin{abstract}
In order to overcome the difficulty of optimizing molecular geometry using quantum Monte Carlo methods, we introduce various approximations to the exact force expectation value. We follow Pulay's suggestion [Mol. Phys. 17, 153 (1969)] to correct the Hellmann-Feynman estimator by introducing the contributions due to the changes in the wave function with respect to the nuclear positions. When used in conjunction with energy-optimized explicitly correlated trial wave functions for $\mathrm{H}_{2}$ and $\mathrm{LiH}$, these approximations appear to yield accurate forces using both the variational and diffusion Monte Carlo methods. Also, the accuracy of the second-order estimate of the Hellmann-Feynman force estimator was investigated employing our energy-optimized trial wave functions, and an erratic behavior was uncovered for some of the studied bond lengths. The additional computational cost required to compute the corrections to the Hellmann-Feynman estimator was found to be only a small fraction of the cost for a simple mean energy calculation. The same approach could be exploited also in computing the derivative of other energy-dependent quantum-mechanical observables. (C) 2003 American Institute of Physics.
\end{abstract}

[DOI: $10.1063 / 1.1562605]$

\section{INTRODUCTION}

In recent years, the quantum Monte Carlo (QMC) methods have been shown to be a powerful tool for solving the Schrödinger equation. ${ }^{1-3} \mathrm{Up}$ to now, QMC techniques have been successfully applied to the calculation of ground-state energies and energy-related properties of a variety of atoms, molecules, and clusters, providing some of the most accurate calculations to date. Despite these remarkable results, the calculation of other important physical properties, such as equilibrium geometries, potential energy surfaces, and vibrational frequencies, has not yet been satisfactorily addressed in this framework. The calculation of such quantities requires the accurate evaluation of the derivative of the energy with respect to a parameter (e.g., the nuclear positions in the case of the internuclear forces), which is known to be very challenging in QMC. The root of the difficulty lies in the stochastic nature of the method itself, always producing an expectation value with a statistical error. This degrades the

${ }^{a)}$ Present address: Dipartimento di Scienze Chimiche, Fisiche e Matematiche, Universitá dell'Insubria, via Lucini 3, 22100 Como, Italy. Electronic mail: mose@ fis.unico.it

${ }^{b)}$ Present address: Central Chemistry Laboratory, Department of Chemistry, University of Oxford, South Parks Road, Oxford, OX1 3QH, United Kingdom. Electronic mail: massimo.mella@chem.ox.ac.uk

${ }^{c)}$ Electronic mail: rappe@sas.upenn.edu computed precision of differences between two similar mean values, as well as the calculation of expectation values whose variance is not bounded.

The Hellmann-Feynman theorem (HFT) provides a very simple and compact expression for evaluating the energy gradient. ${ }^{4,5}$ However, in the past, the use of the HFT for computing forces in QMC was discouraged because of the uncontrolled statistical fluctuations associated with its bare force estimator. A few attempts to circumvent this difficulty were carried out by means of the correlated sampling procedure,${ }^{6-11}$ where the energy difference between two distinct system geometries is directly computed in a single simulation. Unfortunately, the accuracy and precision of the correlated sampling degrades rapidly upon increase of the difference between the two geometries. This difficulty is reduced if the trial wave function for all the geometries different from the reference one is reoptimized, and a "warp" coordinate transformation is used. ${ }^{9,11}$ However, the computational cost needed to compute a $D$-dimensional gradient is, at least, $D+1$ times the one for a single energy estimate, while the cost for the Hessian matrix scales as $D(D+1) / 2$. Obviously, this could become a serious drawback when studying systems containing more than two atoms. Moreover, the scaling properties of the computational cost with respect to the 
atomic number $Z$ of the atoms in the molecule is hard to estimate.

Very recently, a general and effective solution to this problem has been proposed by Assaraf and Caffarel, ${ }^{12}$ who have shown how to remove the pathological part responsible for the infinite variance of the Hellmann-Feynman estimator by using a renormalized operator. In our view, this advancement represents an important step toward accurate force calculations in QMC for systems containing more than two atoms, since it allows for the simultaneous computation of all the gradient components in a single run, and without the burden to optimize $D+1$ wave functions.

This advantage notwithstanding, it should be pointed out that the renormalized Hellmann-Feynman estimator has the drawbacks of not being a zero variance method, and of requiring a computational effort that scales linearly with the atomic number to reach a chosen statistical accuracy. ${ }^{11}$

Moreover, the HFT holds only for the exact eigenfunction of the Hamiltonian operator, or if the special requirement of having all the parameters optimized minimizing the total energy is met by the approximate wave functions (see Sec. II for a discussion). In order to illustrate this point and to introduce our notation, we briefly review the full expression of the force vector in the variational Monte Carlo (VMC) method, where the approximate wave function $\Psi_{T}$ rather than the exact eigenfunction $\Phi_{0}$ is used. Following Pulay's work, ${ }^{13}$ the exact derivative of the VMC energy versus $\mathbf{R}$ (the nuclear positions) can be expressed as

$$
\langle F\rangle_{\mathrm{TOT}}^{\mathrm{VMC}}=\langle F\rangle_{\mathrm{HFT}}^{\mathrm{VMC}}+\langle F\rangle_{\Psi, c}^{\mathrm{VMC}},
$$

where

$$
\langle F\rangle_{\mathrm{HFT}}^{\mathrm{VMC}}=-\frac{\left\langle\Psi_{T}\left|\nabla_{\mathbf{R}} V\right| \Psi_{T}\right\rangle}{\left\langle\Psi_{T} \mid \Psi_{T}\right\rangle}
$$

is the usual HFT estimator, and $\langle F\rangle_{\Psi, c}^{\mathrm{VMC}}$ depends on the variation of $\Psi_{T}$ with respect to the nuclear positions and variational parameters. This term can be exactly decomposed into two terms

$$
\langle F\rangle_{\Psi, c}^{\mathrm{VMC}}=\langle F\rangle_{\Psi}^{\mathrm{VMC}}+\langle F\rangle_{c}^{\mathrm{VMC}}
$$

which read

$$
\begin{aligned}
\langle F\rangle_{\Psi}^{\mathrm{VMC}}=-2 \frac{\left\langle\frac{\partial \Psi_{T}}{\partial \mathbf{R}}|H| \Psi_{T}\right\rangle}{\left\langle\Psi_{T} \mid \Psi_{T}\right\rangle} \\
\\
+2\langle E\rangle_{\mathrm{VMC}} \frac{\left\langle\frac{\partial \Psi_{T}}{\partial \mathbf{R}} \mid \Psi_{T}\right\rangle}{\left\langle\Psi_{T} \mid \Psi_{T}\right\rangle},
\end{aligned}
$$

and

$$
\langle F\rangle_{c}^{\mathrm{VMC}}=-\sum_{i} \frac{\partial c_{i}}{\partial \mathbf{R}} \frac{\partial\langle E\rangle_{\mathrm{VMC}}}{\partial c_{i}} .
$$

Here, $\langle E\rangle_{\mathrm{VMC}}$ is the energy expectation value for the trial wave function, $\nabla_{\mathbf{R}}$ is the gradient with respect to the nuclear coordinates $\mathbf{R}$, and $H$ is the usual Born-Oppenheimer Hamiltonian for the system. In Eq. (4), the partial derivatives of $\Psi_{T}$ with respect to the nuclear positions act only on the part of the trial wave function that explicitly depends on $\mathbf{R}$, i.e., the atomic basis function centers and the correlation factor if any electron-nuclear term is present. The action of $\nabla_{\mathbf{R}}$ on the parameters that only indirectly couple with the nuclear positions (e.g., the coefficients of the molecular orbitals) is rewritten in Eq. (5) by exploiting the chain rule. The $\partial\langle E\rangle_{\mathrm{VMC}} / \partial c_{i}$ terms are the partial derivatives of the mean energy with respect to the set of the variational parameters $c \equiv\left\{c_{i}\right\}$, while the $\partial c_{i} / \partial \mathbf{R}$ are the partial derivatives of these parameters with respect to the nuclear coordinates $\mathbf{R}$.

By virtue of Eqs. (1) and (3), the total force has been separated into three well-defined contributions. Following Eqs. (1)-(5), it is easy to recognize that the HFT formula [Eq. (2)] provides only an approximation to the exact analytic result. Therefore, as far as approximate wave functions are concerned, use of Eq. (2) could result in poor estimates of the energy gradient unless $\Psi_{T}$ is exceedingly accurate. Results using the simple renormalized HFT estimator, i.e., not including $\langle F\rangle_{\Psi, c}^{\mathrm{VMC}}$, were given by Assaraf and Caffarel for $\mathrm{H}_{2}, \mathrm{LiH}, \mathrm{Li}_{2}$, and $\mathrm{C}_{2}{ }^{12}$ at the experimental equilibrium distances. At the $\langle F\rangle_{\mathrm{HFT}}^{\mathrm{VMC}}$ level the computed forces clearly showed a bias, that we interpret as due to some deficiency of the employed wave functions. To partially account for these shortcomings, Assaraf and Caffarel employed the diffusion Monte Carlo (DMC) method together with the second-order estimate (SOE) approximation ${ }^{14}$

$$
\langle F\rangle_{\mathrm{HFT}}^{\mathrm{SOE}} \simeq 2\langle F\rangle_{\mathrm{HFT}}^{\mathrm{DMC}}-\langle F\rangle_{\mathrm{HFT}}^{\mathrm{VMC}},
$$

to correct the electronic density and to evaluate forces in the framework of fixed node (FN) DMC. Although the computed SOE results improved substantially, this procedure, relying on an almost complete error cancellation, is strongly dependent on the quality of the employed trial wave function, sometimes overcorrecting the VMC results. ${ }^{15}$

At this point, one might argue that use of very accurate trial wave functions or densities should be expected to provide accurate, although not exact, results. Therefore, the more accurately the trial wave function approximates the exact one, the closer the agreement between the exact and the HFT estimator should be. At first glance, this expectation seems to be reasonable, but, to our knowledge, it has never been subjected to a systematic investigation within the QMC framework in order to clarify what accuracy can be expected from a given quality of the trial wave function. In this respect it is mandatory to stress that the optimization of a trial wave function in QMC (i.e., the procedure used to select the wave function parameters in order to better approximate an eigenfunction) is a procedure subject to stochastic noise and to the presence of multiple local minima in the parameter space. This is true especially when one chooses to minimize the variance of the local energy $H \Psi_{T} / \Psi_{T}$ instead of the energy, as clearly shown in Ref. 16. There, the authors found that iteratively optimizing the variance of the local energy gave erratic results even for as small a system as $\mathrm{He}_{3}$, and proposed various robust estimators of the quality of a model wave function as a possible cure for this. Although subsequent application by one of us ${ }^{17}$ of these new estimators to more common electronic structure problems showed consis- 
tently better results (i.e., lower energy) than the optimization of the variance of the local energy, a significant stochastic noise was still present. We expect that this finding may also influence the computed force values, for which the accuracy of the trial wave function seems to be an important issue. Therefore, we tried to eliminate the stochastic noise as much as possible, directly optimizing the mean energy using long variational runs. ${ }^{18}$

In this paper, our main aim is to address the issue of how accurately one can compute the forces by means of a different approximate QMC approach, stressing how this knowledge is important with respect to many possible QMC applications. In our view, whereas the work of Assaraf and Caffarel efficiently solved the problem of the unbounded variance of the force estimator, the two major sources of inaccuracy, i.e., the $\langle F\rangle_{\Psi}^{\mathrm{VMC}}$ and $\langle F\rangle_{c}^{\mathrm{VMC}}$ have not been satisfactorily dealt with. As to the $\langle F\rangle_{c}^{\mathrm{VMC}}$ contribution [Eq. (5)], this can be eliminated by energy optimizing the $c$ parameters, so that the partial derivatives $\left[\partial\langle E\rangle_{\mathrm{VMC}}\right] / \partial c_{i}$ have values close to zero. The $\langle F\rangle_{\Psi}^{\mathrm{VMC}}$ term can be formulated analytically and computed accurately in a computational efficient way. In this paper, we demonstrate that merging energy optimization with the exact computation of $\langle F\rangle_{\Psi}^{\mathrm{VMC}}$ leads to very accurate force values with only a fractional increase of the total simulation time.

The outline of the paper is as follows: In Sec. II, we introduce the theoretical and methodological details relevant for the present study. Section III is devoted to presenting and discussing the numerical results obtained for $\mathrm{H}_{2}$ and $\mathrm{LiH}$ as test systems. Finally, Sec. IV contains our conclusions and some prospect for future developments.

\section{THEORY AND METHODS}

It is well known, while the HFT holds for an exhaustively energy-optimized wave function (i.e., a wave function whose variational parameters have been all optimized by minimizing the energy expectation value), the HFT is not exact for QMC trial functions obtained by the common variance minimization scheme. This can be easily demonstrated at the VMC level by considering the analytic energy gradient, Eq. (1). As already mentioned, and well known in the field of $a b$ initio calculations, $\langle F\rangle_{\Psi, c}^{\mathrm{VMC}}$ must be added to $\langle F\rangle_{\mathrm{HFT}}^{\mathrm{VMC}}$ to account for the dependence of the trial wave function on the nuclear coordinates. For sake of convenience, this term was split into two terms in Eq. (4) considering a wave function which contains some parameters that depend directly on the nuclear coordinates (e.g., an atom center basis set or an electron-nucleus Jastrow factor) and other parameters that only depend indirectly.

Although this choice is very natural and convenient in treating molecular systems, in principle, there are no restrictions on the choice of the functional form of the trial wave function. Taking advantage of this freedom, we may also write a trial wave function that does not depend on the nuclear positions directly at all, or, even better, where the atomic basis centers would be considered as variational parameters. As a consequence, the term $\langle F\rangle_{\Psi}^{\mathrm{VMC}}$ would be now included in $\langle F\rangle_{c}^{\mathrm{VMC}}$. Setting $\partial\langle E\rangle_{\mathrm{VMC}} / \partial c_{i}=0$ for each $i$, an equality that holds only for the exact or for a fully energyoptimized wave function, we obtain $\langle F\rangle_{\Psi, c}^{\mathrm{VMC}}=0$, meaning that Eq. (1) and Eq. (2) give the same result.

Unfortunately, obtaining a fully energy-optimized wave function represents a complicated and expensive task for all but the simplest systems, especially when explicitly correlated wave functions are concerned. As a direct consequence, this fact forces us either to optimize a reduced set of parameters or to explicitly compute the correction in Eq. (3). This is commonly carried out in standard ab initio methods by means of the coupled perturbed Hartree-Fock procedure that provides the derivatives of the $c_{i}$ with respect to the nuclear coordinates. This task is not straightforward in QMC, so that a different route must be followed.

In the present work, various levels of approximation are brought to bear on this task with the aim of determining which one delivers enough accuracy and computational efficiency to represent a practical scheme for evaluating accurate forces in QMC.

Before proposing different approximate schemes for evaluating Eq. (1), it is necessary to introduce the general analytical form of the wave functions used. In order to provide a useful benchmark for further calculations, we choose to use the traditional atom-centered wave functions built as $\alpha$ and $\beta$ electron orbital determinants multiplied by the symmetric Jastrow factor proposed by Schmidt and Moskowitz. ${ }^{19,20}$ Whereas the starting molecular orbitals are obtained by means of a standard SCF procedure, the Jastrow parameters are obtained by Newton-Raphson energy minimization. ${ }^{18}$ This model wave function represents the starting point for our investigation of forces. The crudest approximation of forces (VMC-HFT) is made by computing the expectation value of Eq. (2) in VMC, i.e., computing the mean value of the Hellmann-Feynman estimator. As a second approximation, we discard the $\langle F\rangle_{c}^{\mathrm{VMC}}$ term in Eq. (3) approximating the force as a sum of HFT and $\langle F\rangle_{\Psi}^{\mathrm{VMC}}$ terms within VMC (VMC-TOT). Apart from the complication of writing down the explicit form of the equations, ${ }^{21}$ this term is straightforward to compute. Moreover, since many of the quantities needed for its computation are already necessary for standard energy calculation, the increase in the computational cost should be rather limited. The choice of discarding the effect of the dependency of the molecular orbital coefficients on the nuclear position (note we energy optimize the parameters of the Jastrow term) is based on the fact that they should be already close to their optimal values, although obtained in a standard SCF technique. This idea is supported by the small energy improvement obtained by Filippi and Fahy ${ }^{22}$ in reoptimizing the orbitals after multiplying the determinants by a Jastrow factor, and by the computational evidence obtained by two of us ${ }^{21}$ analytically computing the $\partial\langle E\rangle_{\mathrm{VMC}} / \partial c_{i}$. However, this approximation is not mandatory and can be relaxed, optimizing the $c$ 's by means of the Newton-Raphson method ${ }^{21}$ so that all their partial derivatives of the energy are zero. Then, the procedure is formally exact.

In order to remove some of the inaccuracy in the force values due to the limited flexibility of the analytical form of the chosen $\Psi_{T}$, we also compute FN-DMC force estimates 
using our VMC wave function as guiding function. Calculation of the force within the FN-DMC framework is more cumbersome than in the VMC case due to the necessity of computing the derivatives of the exact fixed-node wave function $\Phi_{0}$ with respect to the nuclear coordinates. ${ }^{23}$ This dependency comes from both the nuclear position and the changes in the nodal surfaces of the wave function. Although a formally exact scheme to estimate this quantity has been recently proposed, ${ }^{24}$ it seems to be plagued by large statistical noise. So, to avoid this problem, we approximate such derivatives as proposed by Barnett et al., ${ }^{25}$ writing

$$
\frac{\partial \Phi_{0}}{\partial \mathbf{R}} \cong \Phi_{0}\left(\frac{\partial \Psi_{T} / \partial \mathbf{R}}{\Psi_{T}}\right) .
$$

The resulting FN-DMC force estimator is therefore obtained by differentiating the mixed estimator $\left\langle\Phi_{0}|H| \Psi_{T}\right\rangle$, and reads

$$
\langle F\rangle_{\mathrm{TOT}}^{\mathrm{DMC}} \cong\langle F\rangle_{\mathrm{HFT}}^{\mathrm{DMC}}+\langle F\rangle_{\Psi}^{\mathrm{DMC}},
$$

where

$$
\langle F\rangle_{\mathrm{HFT}}^{\mathrm{DMC}}=-\frac{\left\langle\Phi_{0}\left|\nabla_{\mathbf{R}} V\right| \Psi_{T}\right\rangle}{\left\langle\Phi_{0} \mid \Psi_{T}\right\rangle},
$$

and

$$
\begin{aligned}
\langle F\rangle_{\Psi}^{\mathrm{DMC}}= & -\frac{\left\langle\Phi_{0}\left(\partial \Psi_{T} / \partial \mathbf{R}\right) / \Psi_{T}|H| \Psi_{T}\right\rangle}{\left\langle\Phi_{0} \mid \Psi_{T}\right\rangle} \\
& +\langle E\rangle_{0} \frac{\left\langle\Phi_{0} \mid\left(\partial \Psi_{T} / \partial \mathbf{R}\right)\right\rangle}{\left\langle\Phi_{0} \mid \Psi_{T}\right\rangle} .
\end{aligned}
$$

Similarly to the VMC case, we name DMC-HFT the force estimate obtained by means of Eq. (9), and DMC-TOT the one computed using also Eq. (10). Notice that in Eq. (8) we did not include the contributing coming from the change of the VMC wave function variational parameters with the molecular geometry. One should bear in mind that a similar term would be present even if the exact quantum-mechanical expectation value $\left\langle\Phi_{0}|H| \Psi_{0}\right\rangle$ were used instead of the mixed estimator, due to the changes in the nodal location if any is present. This term is complicated to compute and is expected to be negligible for DMC force calculations as in the VMC case. On the other hand, the term $\langle F\rangle_{\Psi}$ is expected to be vital in order to obtain reliable force estimates.

Following the route we outlined previously, we investigate the accuracy of the four approximations in predicting the forces for the molecules $\mathrm{H}_{2}$ and $\mathrm{LiH}$ within the VMC and FN-DMC frameworks. We can accurately compute the energy expectation values of these systems for different molecular geometries, thus obtaining accurate potential energy surfaces (PES). Moreover, these systems possess two completely different charge distributions (i.e., $\mathrm{H}_{2}$ is nonpolar, while $\mathrm{LiH}$ is almost completely ionic at the equilibrium distance), and highly accurate force values are available for $\mathrm{H}_{2}{ }^{30}$ so that a thorough comparison is possible. Therefore, these systems are ideal candidates for our investigation.

For each molecule, five different bond lengths around the experimental equilibrium distance were considered, namely $95 \%, 97.5 \%, 100 \%, 102.5 \%$, and $105 \%$ of the ex- perimental bond length. These are 1.400 bohr for $\mathrm{H}_{2}$ and 3.015 bohr for LiH. These bond distances were specifically chosen to investigate the region around the minimum energy configuration. The determinants were built starting from restricted SCF wave functions of the DZ (TZ2P) quality for $\mathrm{H}_{2}$ (LiH). To begin our investigation, we optimized for every nuclear distance a Jastrow factor for $\mathrm{H}_{2}$ and $\mathrm{LiH}$, composed by 14 and 30 terms, respectively. These two factors contain for each atom the same terms used in the 9- and 17-term expansions for a single atom in Ref. 19. The Jastrow part of the wave functions corresponding to each molecular geometry were optimized following the procedure described in Ref. 18. This method relies on the use of the Newton secondorder approximation to minimize the VMC energy expectation value with respect to a chosen set of variational parameters. Because of its generality, this approach can be applied to the determinant parameters as well as the parameters belonging to the Jastrow factor. We emphasize that energy optimizing the Jastrow parameters reduces the dependency of the forces on the variation of the wave function parameters, since a subset of their partial derivatives is then equal to zero. Also, energy-optimized trial wave functions seem to yield more accurate properties than variance-optimized ones. This idea is suggested both from theoretical analysis ${ }^{26}$ and empirical numerical evidences. ${ }^{27-29}$ The tables of all the optimized trial wave function parameters are available from the authors upon request.

In the following, we will name JAST the trial wave function obtained employing this optimization strategy. As a starting guess for the optimization procedure, we used a unit Jastrow factor, i.e., with zeros as initial parameter values. Once optimized, the resulting wave functions were used to compute both $\langle F\rangle_{\mathrm{HFT}}$ and $\langle F\rangle_{\mathrm{HFT}}+\langle F\rangle_{\Psi}$ averages in VMC and FN-DMC. In order to avoid the problem related to the infinite variance of the HFT estimator, we modified Eq. (2) and Eq. (9) as suggested by Assaraf and Caffarel. ${ }^{12}$

\section{RESULTS AND DISCUSSION}

VMC and FN-DMC force estimates for $\mathrm{H}_{2}$ are collected in Table I and shown in Fig. 1. Table I also reports the corresponding VMC and FN-DMC energy values, accurate force estimates obtained from Ref. 30, and correlation energy percentages. The $\langle F\rangle_{\text {Exact }}$ force values were obtained by fitting with a second-order polynomial the highly accurate forces computed in Ref. 30 in the range 1.30-1.50 bohr. The fitted polynomial was found to differ from the fitted values by less than 0.0001 hartree/bohr, so indicating the global accuracy of the values shown in Table I. The correlation energy percentages were obtained using the Hartree-Fock limit taken from Ref. 31, and the FN-DMC energies computed in this work. These last ones are to be considered statistically exact, since $\mathrm{H}_{2}$ has a ground state with no nodes and we carefully checked the time-step bias to be smaller than the statistical error.

Our optimized wave functions were found to recover a considerable fraction of the correlation energy at a variational level, approximately 92\%. Given this high level of total energy accuracy, let us focus our attention on the VMC 
TABLE I. VMC and FN-DMC expectation values of the forces and energies for $\mathrm{H}_{2}$ over a JAST optimized function. All the VMC and DMC simulations were carried out sampling the same number of total configurations. $\langle F\rangle_{\mathrm{TOT}}$ indicates $\langle F\rangle_{\mathrm{HFT}}+\langle F\rangle_{\Psi} \cdot\langle F\rangle_{\text {Exact }}$ from Ref. 30. $\mathrm{CE}_{\%}$ is the correlation energy percentage. Forces are in hartree/bohr, energies are in hartree, distance in bohr.

\begin{tabular}{|c|c|c|c|c|c|}
\hline$R$ & 1.470 & 1.435 & 1.400 & 1.365 & 1.330 \\
\hline$\langle E\rangle^{\mathrm{VMC}}$ & $-1.17010(2)$ & $-1.17107(2)$ & $-1.17143(2)$ & $-1.17128(2)$ & $-1.17052(2)$ \\
\hline $\mathrm{CE}_{\%}$ & 91.4 & 92.2 & 92.1 & 93.2 & 92.7 \\
\hline$\langle E\rangle^{\mathrm{DMC}}$ & $-1.17364(2)$ & $-1.17425(2)$ & $-1.17444(3)$ & $-1.17417(2)$ & $-1.17344(2)$ \\
\hline$\langle F\rangle_{\mathrm{HFT}}^{\mathrm{VMC}}$ & $-0.01402(6)$ & $-0.02642(5)$ & $-0.03685(5)$ & $-0.04872(5)$ & $-0.06620(5)$ \\
\hline$\langle F\rangle_{\Psi}^{\mathrm{VMC}}$ & $0.04223(2)$ & $0.04372(2)$ & $0.04081(3)$ & $0.03827(1)$ & $0.04062(2)$ \\
\hline$\langle F\rangle_{\mathrm{TOT}}^{\mathrm{VMC}}$ & $0.02821(6)$ & $0.01729(5)$ & $0.00395(6)$ & $-0.01044(5)$ & $-0.02558(5)$ \\
\hline$\langle F\rangle_{\mathrm{HFT}}^{\mathrm{DMC}}$ & $0.0038(1)$ & $-0.0080(1)$ & $-0.0192(1)$ & $-0.0321(1)$ & $-0.0403(1)$ \\
\hline$\langle F\rangle_{\Psi}^{\mathrm{DMC}}$ & $0.02077(1)$ & $0.02151(1)$ & $0.02003(2)$ & $0.01892(1)$ & $0.01843(2)$ \\
\hline$\langle F\rangle_{\mathrm{TOT}}^{\mathrm{DMC}}$ & $0.0245(1)$ & $0.0135(1)$ & $0.0008(1)$ & $-0.0132(1)$ & $-0.0218(1)$ \\
\hline$\langle F\rangle_{\text {Exact }}$ & $0.02285(1)$ & $0.01200(1)$ & $-0.00040(1)$ & $-0.01438(1)$ & $-0.02992(1)$ \\
\hline
\end{tabular}

estimates first. It is clear that, even for our highly accurate wave functions, the difference between $\langle F\rangle_{\mathrm{HFT}}^{\mathrm{VMC}}$ and $\langle F\rangle_{\mathrm{TOT}}^{\mathrm{VMC}}$ is of the same order of magnitude as the force average itself. The conditions for the HFT are clearly violated. It is interesting to note that, regardless of the molecular geometry, we find for this system $\langle F\rangle_{\Psi}$ to be approximately constant, 0.041 hartree/bohr. As a consequence, the $\langle F\rangle_{\mathrm{TOT}}^{\mathrm{VMC}}$ values are shifted upwards with respect to the $\langle F\rangle_{\mathrm{HFT}}^{\mathrm{VMC}}$ by similar amounts. This shift has a profound impact on the calculation of the equilibrium geometry as shown in Fig. 1. Here, the intersection of the two fitted lines with the $\langle F\rangle=0$ axis provides an estimate for the equilibrium geometry. This yields $1.506(7)$ bohr for $\langle F\rangle_{\mathrm{HFT}}^{\mathrm{VMC}}$ and 1.393(2) bohr for $\langle F\rangle_{\mathrm{TOT}}^{\mathrm{VMC}}$, differing, respectively, by $7 \%$ and $0.5 \%$ from the experimental bond length. Also, the value obtained from $\langle F\rangle_{\mathrm{HFT}}^{\mathrm{VMC}}$ is clearly in contrast with the VMC PES behavior (see Fig. 2), whose minimum, as obtained by quadratic fitting, is $1.392(2)$ bohr. Conversely, the TOT estimator accurately agrees with the fitted PES result, giving an estimate of the equilibrium distance in statistical agreement with it. In addition, the use of $\langle F\rangle_{\mathrm{TOT}}^{\mathrm{VMC}}$ seems to have the important effect of reducing the statistical error of the computed equilibrium distance, as can be seen comparing its standard deviation for VMC-HFT, $0.007 \mathrm{bohr}$, and for VMC-TOT, 0.002 bohr. This outcome is due to a less noisy, i.e., more linear, behavior of the com-

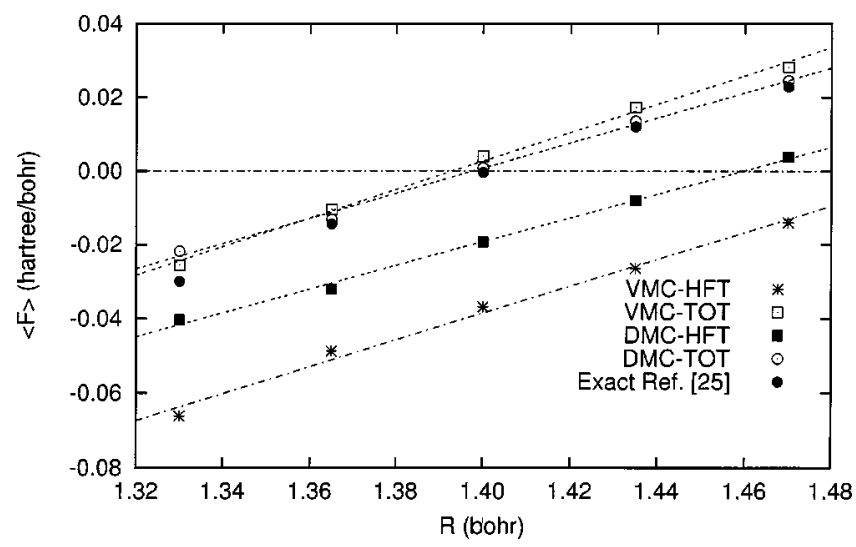

FIG. 1. Exact (see the text), VMC, and DMC force estimates using JAST wave functions for $\mathrm{H}_{2}$. Quantities in a.u. Statistical errors (not shown) are smaller than the plotted symbol. puted force with respect to the nuclear distance, as seen in Fig. 1. This suggests that the $\langle F\rangle_{\text {TOT }}^{\mathrm{VMC}}$ estimator is able, at least partially, to correct for small shortcomings of the wave functions.

Turning now to the FN-DMC forces, it clearly appears that the HFT estimates, although significantly improved with respect to their VMC counterparts, still show some drawbacks due to not accounting for the changes in the wave function. Similarly to the VMC case, adding the term $\langle F\rangle_{\Psi}$ significantly changes the force with respect to the HFT estimate. We also note that $\langle F\rangle_{\Psi}^{\mathrm{DMC}}$ appears to be approximately constant similarly to the VMC case, regardless of the molecular geometry. Although this value is approximately half the value we encountered discussing the VMC averages (roughly 0.02 hartree/bohr versus 0.041 hartree/bohr), this term causes significant change. The Hellmann-Feynman estimates have, once again, lower values than their total counterparts. The internuclear equilibrium distance predicted by the HFT estimator is quite far from the PES value even in DMC. These are, respectively, 1.459(3) and 1.404(3) bohr, the last one being obtained by a second-order polynomial fit of the DMC energies. Conversely, the TOT force estimator provides the more accurate result of 1.398(2) bohr, which is also close to that obtained by fitting $\langle F\rangle_{\mathrm{TOT}}^{\mathrm{VMC}}[1.393(2)$ bohr].

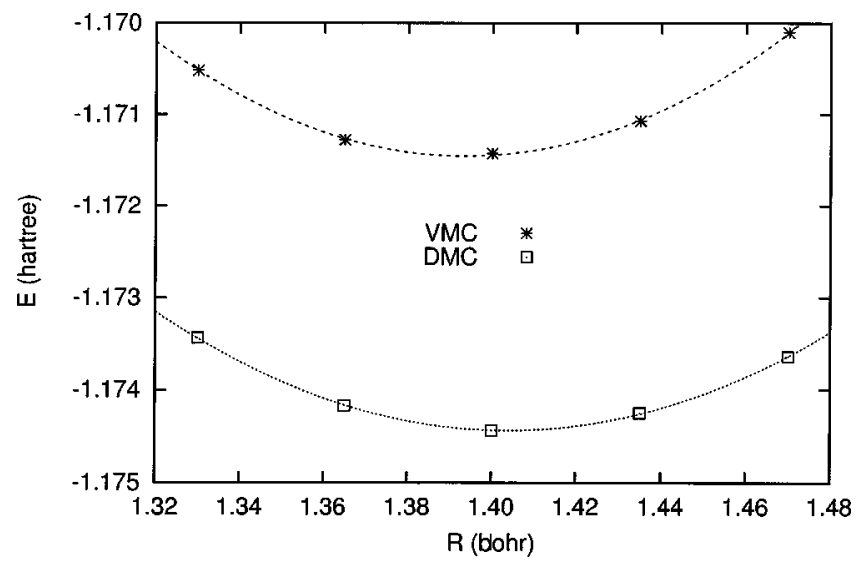

FIG. 2. VMC and DMC energies using JAST wave functions for $\mathrm{H}_{2}$. Quantities in a.u. Statistical errors (not shown) are smaller than the plotted symbols. 
TABLE II. VMC and FN-DMC expectation values of the forces and energies for LiH over a JAST optimized function. All the VMC and DMC simulations were carried out sampling the same number of total configurations. $\langle F\rangle_{\mathrm{TOT}}$ indicates $\langle F\rangle_{\mathrm{HFT}}+\langle F\rangle_{\Psi} . \mathrm{CE}_{\%}$ is the correlation energy percentage. Forces are in hartree/bohr, energies are in hartree, distance in bohr.

\begin{tabular}{lccccc}
\hline \hline \multicolumn{1}{c}{$R$} & 3.16575 & 3.09037 & 3.0150 & 2.939625 & 2.86425 \\
\hline$\langle E\rangle^{\mathrm{VMC}}$ & $-8.06234(8)$ & $-8.06298(8)$ & $-8.06348(8)$ & $-8.06281(6)$ & $-8.06252(6)$ \\
$\mathrm{CE}_{\%}$ & 91.0 & 91.35 & 93.1 & 91.1 & 92.0 \\
$\langle E\rangle^{\mathrm{DMC}}$ & $-8.06975(7)$ & $-8.07014(9)$ & $-8.07027(6)$ & $-8.07000(7)$ & $-8.06903(11)$ \\
$\langle F\rangle_{\mathrm{HFT}}^{\mathrm{VMC}}$ & $0.02384(7)$ & $0.01344(7)$ & $0.0090(1)$ & $0.00487(7)$ & $-0.00335(8)$ \\
$\langle F\rangle_{\Psi}^{\mathrm{VMC}}$ & $-0.01583(10)$ & $-0.00989(7)$ & $-0.01108(10)$ & $-0.01174(6)$ & $-0.01103(13)$ \\
$\langle F\rangle_{\mathrm{TOT}}^{\mathrm{VMC}}$ & $0.00800(10)$ & $0.00356(10)$ & $-0.00208(10)$ & $-0.00687(9)$ & $-0.01438(16)$ \\
$\langle F\rangle_{\mathrm{HFT}}^{\mathrm{DMC}}$ & $0.01581(13)$ & $0.00863(18)$ & $0.0043(10)$ & $-0.00037(13)$ & $-0.00732(19)$ \\
$\langle F\rangle_{\Psi}^{\mathrm{DMC}}$ & $-0.00736(5)$ & $-0.00394(6)$ & $-0.00464(4)$ & $-0.00493(4)$ & $-0.00420(8)$ \\
$\langle F\rangle_{\mathrm{TOT}}^{\mathrm{DMC}}$ & $0.00845(14)$ & $0.00469(18)$ & $-0.00024(14)$ & $-0.00531(14)$ & $-0.01152(21)$ \\
\hline \hline
\end{tabular}

So, while the bond length estimate from DMC-HFT force is off by $4 \%$ from the exact value, the DMC-TOT one is statistically exact, and in excellent agreement with the PES minimum.

Comparing our results with the $\langle F\rangle_{\text {Exact }}$ makes evident the large improvement of the TOT values with respect to the HFT ones for both VMC and DMC over the entire bond length range. Interestingly, at any bond length we found the differences between the $\langle F\rangle_{\mathrm{TOT}}^{\mathrm{VMC}}$ and the corresponding $\langle F\rangle_{\mathrm{TOT}}^{\mathrm{DMC}}$ averages ranging from 0.00315 to 0.0036 hartree/ bohr, hence an order of magnitude smaller than the correction $\langle F\rangle_{\Psi}^{\mathrm{DMC}}$. This finding, together with the valuable improvement in the VMC force estimates due to the addition of the term $\langle F\rangle_{\Psi}^{\mathrm{VMC}}$ to the HFT estimator, suggests the possibility of accurately computing forces without using the FNDMC method, relying on the simpler VMC. This choice would allow the faster decorrelation between different samples that one obtains in VMC to be exploited. This is given by the possibility of using larger time steps in a VMC simulation than in a DMC one, although still using an exact Metropolis scheme. Thus, VMC provides forces with greater statistical accuracy than DMC when performing runs of the same length. This is clearly seen comparing the standard deviations of the VMC-TOT and the DMC-TOT results in

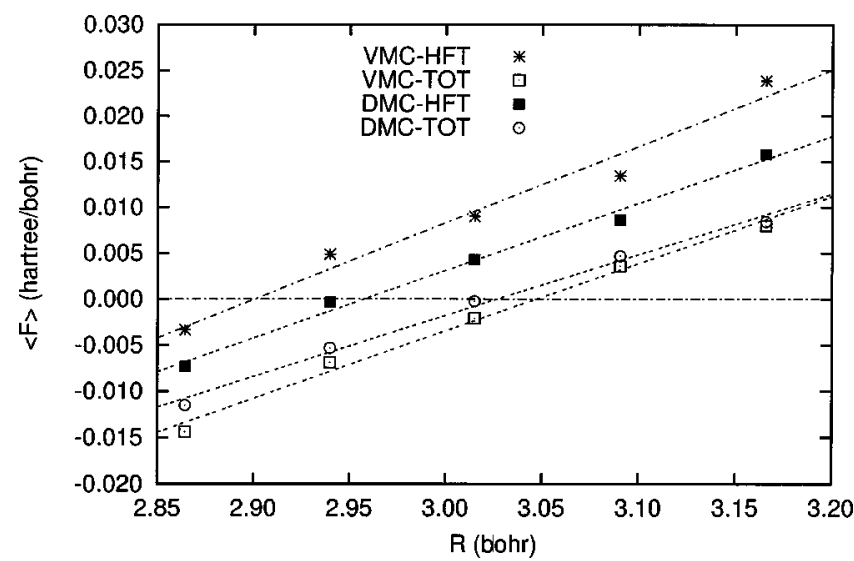

FIG. 3. VMC and FN-DMC force estimates using JAST wave functions for LiH. Quantities in a.u. Statistical errors (not shown) are smaller than the plotted symbols.
Table I, the first usually being half of the second, although simulations sampling the same number of configurations were employed.

Our results for $\mathrm{LiH}$ are summarized in Table II and pictorially shown in Fig. 3. Here, the correlation energy percentages were computed using Hartree-Fock results from Ref. 32. Our JAST optimized wave functions recovered more than $91 \%$ of the correlation energy on the average. The use of $\langle F\rangle_{\mathrm{TOT}}^{\mathrm{VMC}}$ instead of $\langle F\rangle_{\mathrm{HFT}}^{\mathrm{VMC}}$ as force estimator provides very different results, in close similarity to the $\mathrm{H}_{2}$ case. However, $\langle F\rangle_{\Psi}^{\mathrm{VMC}}$ considerably varies on going from the longest to the shortest internuclear distance. For all the geometries considered, the HFT estimates show higher values than the total ones. Once more, this difference is responsible for very different values of the internuclear equilibrium distance, as shown in Fig. 3. Whereas the equilibrium distance predicted by the HFT formula [2.900(16) bohr] underestimates by $4 \%$ the one obtained by fitting the VMC PES [3.015(26) bohr] shown in Fig. 4, a value in better agreement with this one is provided by the TOT estimator [3.043(6) bohr], that is off by only $1 \%$.

Notice that the statistical error of the $\mathrm{LiH}$ equilibrium distances, 0.016 bohr for the VMC-HFT estimate and 0.006 bohr for the VMC-TOT one, are higher than those obtained for $\mathrm{H}_{2}$, respectively, 0.007 and $0.002 \mathrm{bohr}$, owing to a wider

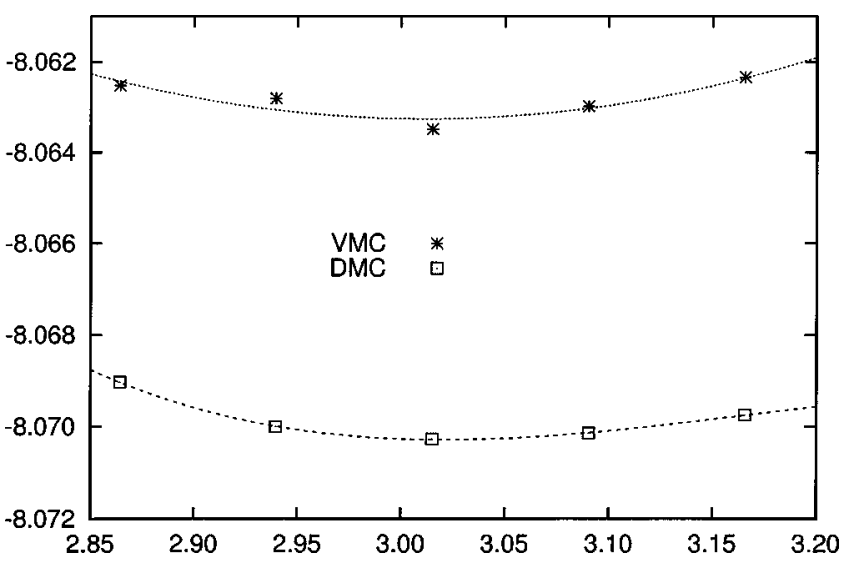

FIG. 4. VMC and FN-DMC LiH potential energy surfaces. Quantities in a.u. Statistical errors (not shown) are smaller than the plotted symbols. 
dispersion of the LiH force averages (see Fig. 3). This dispersion presumably relates to the stochastic noise inherent in the wave function optimization, as well as to the orbital basis set incompleteness. However, similarly to the previous system, the correction $\langle F\rangle_{\Psi}^{\mathrm{VMC}}$ appears to considerably decrease the dispersion of the force values, therefore allowing for a more statistically precise estimate of the equilibrium distance. In Table II and in Fig. 3, we also show the corresponding FN-DMC estimates, for which remarks similar to those for the VMC case could be made. More specifically, the $\langle F\rangle_{\Psi}^{\mathrm{DMC}}$ decreases by a substantial amount the $\langle F\rangle_{\mathrm{HFT}}^{\mathrm{DMC}}$ estimate, concurrently reducing their dispersion. This last effect can be seen either from Fig. 3, or from the statistical error of the computed equilibrium distances. These are 2.958(7) bohr for the HFT case, and 3.026(5) bohr for the TOT one. This latter is in much better agreement than the HFT one with the minimum of the DMC PES [3.041(6) bohr; see Fig. 4] than the HFT one. Note that, in the case of DMC, fitting the force values has provided a result with the same statistical accuracy of that obtained by the PES fitting (about 0.006 bohr). From this point of view, use of the force values instead of the energy ones might seem to make no difference in predicting the equilibrium geometry. However, as far as geometry optimization is concerned, the calculation of the force vector is much more advantageous than that of the sole energy expectation value, since it provides the direction toward the minimum energy configuration. This is a clear advantage in the case of a multidimensional PES for which a systematic exploration of all the degrees of freedom is computationally expensive, if not impossible, to carry out.

At this point it is important to stress that for both $\mathrm{H}_{2}$ and $\mathrm{LiH}$, the VMC-TOT and FN-DMC-TOT equilibrium distance results agree with experiments equally well, the difference being no more than a few times their statistical error. Again, these results suggest that geometry optimization using simply the VMC-TOT method will provide as high accuracy and greater efficiency than DMC-TOT.

As a final test of our $\langle F\rangle_{\mathrm{HFT}}^{\mathrm{VMC}}+\langle F\rangle_{\Psi}^{\mathrm{VMC}}$ approximation, it would be interesting to check whether it may yield even more satisfactory results when more accurate wave functions were used. In order to carry out such a test, we extended the Newton's optimization method to deal also with the determinant parameters, i.e., optimizing the linear coefficients of the molecular orbitals and the exponents of the atomic basis sets. For sake of clarity, in the following we will name DET the optimization procedure where all the wave function parameters, except the atomic orbital centers, were fully energy optimized. Since the optimization of the determinant parameters was found to be quite expensive, we restricted our calculations to the experimental equilibrium geometry for both molecules. Our energy and force results are collected in Table III.

For both the molecules, the reoptimization of the determinant resulted in a small gain in the correlation energy percentages ( $4.6 \%$ for $\mathrm{H}_{2}$, and $1.0 \%$ for $\mathrm{LiH}$ ) with respect to the results shown in Tables I and II. In the case of the hydrogen molecule, the improvement in the correlation energy percentage is larger due to the small size of the DZ basis set used in the calculation. Conversely, the LiH TZP basis set is nearly
TABLE III. VMC and FN-DMC expectation values of the forces and energies for $\mathrm{H}_{2}$ and LiH over DET optimized function. All the VMC and DMC simulations were carried out sampling the same number of total configurations. $\langle F\rangle_{\text {TOT }}$ indicates $\langle F\rangle_{\mathrm{HFT}}+\langle F\rangle_{\Psi} . \mathrm{CE}_{\%}$ is the correlation energy percentage. Quantities in a.u.

\begin{tabular}{lcc}
\hline \hline & $\mathrm{H}_{2}(R=1.400$ bohr $)$ & $\mathrm{LiH}(R=3.0150$ bohr $)$ \\
\hline$\langle E\rangle^{\mathrm{VMC}}$ & $-1.17355(1)$ & $-8.06475(5)$ \\
$\mathrm{CE}_{\%}$ & 96.7 & 94.1 \\
$\langle E\rangle^{\mathrm{DMC}}$ & $-1.17449(3)$ & $-8.0701(1)$ \\
$\langle F\rangle_{\mathrm{H} F \mathrm{VT}}^{\mathrm{VMC}}$ & $-0.00197(8)$ & $0.00492(8)$ \\
$\langle F\rangle_{\Psi}^{\mathrm{VMC}}$ & $0.00326(1)$ & $-0.0069(4)$ \\
$\langle F\rangle_{\mathrm{TMC}}^{\mathrm{VMC}}$ & $0.00129(8)$ & $-0.0019(4)$ \\
$\langle F\rangle_{\mathrm{HFC}}^{\mathrm{DMC}}$ & $-0.0015(2)$ & $0.0020(2)$ \\
$\langle F\rangle_{\Psi}^{\mathrm{DMC}}$ & $0.00158(2)$ & $-0.00220(8)$ \\
$\langle F\rangle_{\mathrm{TOT}}^{\mathrm{DMC}}$ & $0.0001(2)$ & $-0.0002(2)$ \\
$\langle F\rangle_{\mathrm{HFT}}^{\mathrm{SOE}}$ & $-0.0010(2)$ & $-0.0009(2)$ \\
\hline \hline
\end{tabular}

converged to the Hartree-Fock limit. As expected, optimizing the determinant yields a better agreement between the VMC-HFT and VMC-TOT averages with respect to that obtained for the JAST results. For $\mathrm{H}_{2}$ the difference between these estimates is found to be 0.003 64(1) hartree/bohr, approximately $1 / 10$ of the previous value (see Table I for a comparison), while for $\mathrm{LiH}$ this difference $[0.0069(4)$ hartree/bohr] is $1 / 2$ (see Table II). A similar behavior is found also for the DMC estimates, the correction to HFT for $\mathrm{H}_{2}$ $(\mathrm{LiH})$ being $1 / 13(1 / 2)$ of the DMC-JAST one. However, despite the considerable gain, the HFT and the TOT estimates cannot be said to be statistically equivalent. Even in this case, the residual difference suggests that the HFT approaches do not represent a satisfactory measure of the energy gradient. More interestingly, the DET-VMC-TOT and JAST-VMC-TOT forces at the equilibrium distance appear to be in good agreement, especially in the case of $\mathrm{LiH}$, where the optimization of the atomic basis set should play a minor role in defining their values. Adding to these considerations the computational cost required to address the optimization of the determinant, we conclude that one should not expect the HFT estimator to be useful within the VMC and the DMC frameworks for computing forces. On the contrary, its TOT counterpart does not require the trial wave function to be exhaustively optimized, and it can be used efficiently to predict force averages in agreement with the PES behavior and to calculate accurately the internuclear equilibrium distance. This data is also supported by the accurate agreement between the VMC-TOT and DMC-TOT equilibrium distance estimates.

Having probed the overall performance of the VMCTOT and DMC-TOT approximations, the last point that remains to be addressed regards the comparison with the SOE [Eq. (6)] in computing force values. This approach was proposed by Assaraf and $\mathrm{Caffarel}^{12}$ in order to improve the HFT estimate, and indeed they found that $\langle F\rangle_{\mathrm{HFT}}^{\mathrm{SOE}}$ always corrected the VMC and DMC values in the right direction. However, they published results only for the experimental equilibrium distance, so that the ability of the SOE to predict this quantity accurately could not be thoroughly assessed. Also, our wave functions for both $\mathrm{H}_{2}$ and $\mathrm{LiH}$, recovering more correlation energy than the ones employed in Ref. 12, should rep- 
TABLE IV. SOE expectation values of the forces for $\mathrm{H}_{2}$ and $\mathrm{LiH}$ using JAST optimized functions. Quantities in a.u. $\Delta$ is the difference with $\langle F\rangle_{\mathrm{TOT}}^{\mathrm{DMC}}$ taken from Table I and Table II.

\begin{tabular}{lrrrrr}
\hline \hline$R\left(\mathrm{H}_{2}\right)$ & 1.470 & 1.435 & 1.400 & 1.365 & 1.330 \\
\hline$\langle F\rangle_{\mathrm{HFT}}^{\mathrm{SOE}}$ & $0.0216(1)$ & $0.0104(1)$ & $0.0016(1)$ & $-0.0155(1)$ & $-0.0144(1)$ \\
$\Delta$ & $-0.0029(1)$ & $-0.0031(1)$ & $+0.0008(1)$ & $-0.0023(1)$ & $-0.0074(1)$ \\
\hline$R(\mathrm{LiH})$ & 3.16575 & 3.09037 & 3.0150 & 2.939625 & 2.86425 \\
\hline$\langle F\rangle_{\mathrm{HFT}}^{\mathrm{SOE}}$ & $0.0081(2)$ & $0.0038(2)$ & $-0.0004(1)$ & $-0.0056(2)$ & $-0.0113(1)$ \\
$\Delta$ & $-0.0003(2)$ & $-0.0009(3)$ & $0.0002(1)$ & $-0.0003(2)$ & $+0.0002(1)$ \\
\hline \hline
\end{tabular}

resent a better starting point. Our results of the $\langle F\rangle_{\mathrm{HFT}}^{\mathrm{SOE}}$ for both $\mathrm{H}_{2}$ and $\mathrm{LiH}$ are collected in Table IV for the JAST wave functions, and in Table III for the DET wave functions at the equilibrium distance. Also, in Table IV we show $\Delta$ $=\langle F\rangle_{\mathrm{HFT}}^{\mathrm{SOE}}-\langle F\rangle_{\mathrm{TOT}}^{\mathrm{DMC}}$ in order to facilitate the comparison between the two estimates. As previously suggested in Ref. 12, the SOE estimate noticeably improves the HFT force estimate, bringing it in closer agreement with the $\langle F\rangle_{\mathrm{TOT}}^{\mathrm{DMC}}$ one. Although this fact is true for both $\mathrm{H}_{2}$ and $\mathrm{LiH}$, one could note that a better agreement between the two estimators is obtained in the $\mathrm{LiH}$ case. Moreover, for $\mathrm{H}_{2} \Delta$ shows an erratic behavior with an unexpected fall at $1.330 \mathrm{bohr}$, in clear contrast with the smoother behavior of the DMC-TOT estimate. In our view, this finding highlights the strong dependency of the $\langle F\rangle_{\mathrm{HFT}}^{\mathrm{SOE}}$ on the quality of $\Psi_{T}$. The erratic behavior is also evident from the large statistical error of the equilibrium distance, 1.40(1) bohr, computed excluding the aforementioned force value for the shorter distance. Conversely, the equilibrium distance for $\mathrm{LiH}$ computed using the SOE results, 3.032(6) bohr, has a statistical error similar to the DMC-TOT one. Somewhat ironically, the DET-SOE force for $\mathrm{H}_{2}$ shown in Table III does not improve the agreement with the DMC-TOT result, overshooting it at the experimental equilibrium distance. Also, $\langle F\rangle_{\mathrm{HFT}}^{\mathrm{SOE}}$ still differs by more than 4 standard deviation from zero, i.e., the exact value, for the $\mathrm{LiH}$.

At this point, due to the large improvement in the quality of the trial wave function for the hydrogen molecule obtained by optimizing the orbitals, one may argue that this peculiar behavior should not be due to the DZ basis set, but rather to an incomplete error cancellation in the SOE. Here, it is worth citing that a similar misbehavior of SOE was previously pointed out in the QMC literature for many different physical properties. To name a few examples, we cite the calculation of $\langle\delta(r)\rangle$ for electronic ${ }^{33}$ and mixed electronic-positronic ${ }^{34}$ systems, and the dipole moment calculation of $\mathrm{LiH}$ by $\mathrm{Lu}^{36}$ All these findings can be rationalized invoking the results obtained by Sarsa, Schmidt, and Magro. ${ }^{35}$ Employing a formally exact variational path integral procedure to compute expectation values for both model and realistic systems, they found that SOE may give a difference with the exact result similar to the VMC one. The magnitude of this difference seemed to depend strongly on the quality of the trial wave function used in the simulation.

As to the computational cost, a question might be posed about the effort needed to compute the sum $\langle F\rangle_{\mathrm{HFT}}+\langle F\rangle_{\Psi}$ rather than $\langle F\rangle_{\mathrm{HFT}}$ only. Indeed, some authors have implied that this calculation might be computationally expensive or cumbersome because of the mathematical expression of the derivatives involved, ${ }^{37}$ either requiring more CPU time or more simulation steps. In contrast to this conclusion, however, we note that, for both force estimators the same statistical accuracy was achieved (see Tables I and II), and thus the calculation of the total averages did not require longer runs than those needed to compute the HFT values. Obviously the calculation of the total estimator is expected to increase the computational time, because of the presence of the term $\langle F\rangle_{\Psi}$. However, performing variational calculations, we found this additional amount to be small enough (about $10 \%$ of the total time) to guarantee reasonable computational costs.

\section{CONCLUSIONS}

In this work, various approximate estimates of the nuclear forces have been proposed and tested in the framework of the QMC methods. From our results on $\mathrm{H}_{2}$ and $\mathrm{LiH}$, it emerges that computing $\langle F\rangle_{\mathrm{HFT}}+\langle F\rangle_{\Psi}$ at the VMC level, using a $\Psi_{T}$ whose Jastrow factor has been optimized by minimizing the energy, can be an accurate and viable approach to obtain forces. Also, VMC-TOT seems to partially correct for the inaccuracy of $\Psi_{T}$, a feature that every approximate estimator should have. As a consequence, the computed equilibrium distances are in accurate agreement with the experimental ones.

This finding seems to be somehow in mismatch with the results of Ref. 11, where larger differences between VMC and exact equilibrium distances have been found for the firstrow dimers. Here, it is important to stress that the results presented in Ref. 11 were computed without reoptimizing the secondary geometry wave functions; therefore, a systematic error could be expected. Bearing in mind the greater efficiency of VMC with respect to DMC, we believe that the issue of the accuracy of VMC forces deserves further attention.

If greater accuracy is needed, we found the DMC-TOT well suited to tackle the task, giving, overall, an order of magnitude more accurate results in our model systems. As far as the SOE is concerned, although it has been found to substantially improve the HFT force estimate, it shows a more erratic and noisy behavior than the TOT counterpart. Besides, its accuracy appears to be similar to the VMC-TOT one, but its cost is at least double due to the necessity of running a DMC simulation. 
In conclusion, let us remark that use of Eq. (1) is not exclusively restricted to the determination of force averages. Indeed, it can be used instead of the HFT to compute energy derivatives with respect to the nuclear positions whenever these are needed. As examples of possible application we cite the calculation of bond force constants, the Hessian matrix of the energy, and the derivatives of the molecular dipole moment, a quantity closely connected with the infrared absorption intensity.

\section{ACKNOWLEDGMENTS}

This work was supported by the Air Force Office of Scientific Research, Air Force Materiel Command, USAF, under Grant No. F49620-00-1-0170 and the Office of Naval Research, under Grant No. N-00014-00-1-0372.

${ }^{1}$ W. M. C. Foulkes, L. Mitas, R. J. Needs, and G. Rajagopal, Rev. Mod. Phys. 73, 33 (2001).

${ }^{2}$ A. Lüchow and J. B. Anderson, Annu. Rev. Phys. Chem. 51, 527 (2000).

${ }^{3}$ M. Mella, S. Chiesa, D. Bressanini, and G. Morosi, in New Directions in Antimatter Chemistry and Physics, edited by C. M. Surko and F. A. Gianturco (Kluwer Academic, Dordrecht, 2001), p. 235.

${ }^{4}$ H. Hellmann, Einführung in die Quanten Theorie (Deuticke, Leipzig, 1937).

${ }^{5}$ R. P. Feynman, Phys. Rev. 56, 340 (1939).

${ }^{6}$ B. H. Wells, Chem. Phys. Lett. 115, 89 (1985).

${ }^{7}$ C. A. Traynor and J. B. Anderson, Chem. Phys. Lett. 147, 389 (1988).

${ }^{8}$ C. J. Umrigar, Int. J. Quantum Chem., Quantum Chem. Symp. 23, 217 (1989).

${ }^{9}$ C. Filippi and C. J. Umrigar, Phys. Rev. B 61, R16291 (2000)

${ }^{10}$ J. Vrbik and S. M. Rothstein, J. Chem. Phys. 96, 2071 (1991).

${ }^{11}$ C. Filippi and C. J. Umrigar, Recent Advances of Quantum Monte Carlo Methods, Part II, edited by W. A. Lester, Jr., S. M. Rothstein, and M.

Tanaka (World Scientific, Singapore, 2002), pp. 12-29.

${ }^{12}$ R. Assaraf and M. Caffarel, J. Chem. Phys. 113, 4028 (2000).

${ }^{13}$ P. Pulay, Mol. Phys. 17, 153 (1969).
${ }^{14}$ D. M. Ceperley and M. H. Kalos, "Quantum Many-Body Problems," in Monte Carlo Methods in Statistical Physics (Springer, New York, 1979), Vol. 183.

${ }^{15}$ D. Bressanini, M. Mella, and G. Morosi, Phys. Rev. A 57, 1678 (1998).

${ }^{16}$ D. Bressanini, G. Morosi, and M. Mella, J. Chem. Phys. 116, 5345 (2002).

${ }^{17} \mathrm{M}$. Mella, unpublished results. The optimization of the Jastrow factor for the three small systems $\mathrm{He}, \mathrm{Be}$, and $\mathrm{LiH}$ gave a more stable optimization, a smoother converging behavior, and a lower energy when the robust estimators proposed in Ref. 16 were used instead of the variance of the local energy.

${ }^{18}$ X. Lin, H. Zhang, and A. M. Rappe, J. Chem. Phys. 110, 4619 (1999).

${ }^{19}$ K. E. Schmidt and J. W. Moskowitz, J. Chem. Phys. 93, 4172 (1990).

${ }^{20}$ J. W. Moskowitz and K. E. Schmidt, J. Chem. Phys. 97, 3382 (1992).

${ }^{21}$ M. Casalegno and A. M. Rappe (unpublished).

${ }^{22}$ C. Filippi and S. Fahy, J. Chem. Phys. 112, 3523 (2000).

${ }^{23}$ F. Schautz and H.-J. Flad, J. Chem. Phys. 110, 11700 (1999); 112, 4421 (2000).

${ }^{24}$ S. De Palo, S. Moroni, and S. Baroni, http://xxx.sissa.it/abs/ cond-mat/0111486

${ }^{25}$ R. N. Barnett, P. J. Reynolds, and W. A. Lester, Int. J. Quantum Chem. 29, 589 (1986).

${ }^{26}$ H. M. James and A. S. Coolidge, Phys. Rev. 51, 860 (1937).

${ }^{27}$ R. Bianchi, D. Bressanini, P. Cremaschi, M. Mella, and G. Morosi, Int. J. Quantum Chem. 57, 321 (1994).

${ }^{28}$ D. Bressanini, P. Cremaschi, M. Mella, and G. Morosi, in Recent Advances in Quantum Monte Carlo Methods, edited by W. A. Lester, Jr. (World Scientific, Singapore, 1997).

${ }^{29}$ M. Snajdr and S. M. Rothstein, J. Chem. Phys. 112, 4935 (2000).

${ }^{30}$ W. Kolos and L. Wolniewicz, J. Chem. Phys. 7, 2429 (1965).

${ }^{31}$ W. Kolos and C. C. J. Roothaan, Rev. Mod. Phys. 32, 219 (1960).

${ }^{32}$ N. C. Handy, R. J. Harrison, P. J. Knowles, and H. F. Schaefer III, J. Phys. Chem. 88, 4852 (1984).

${ }^{33}$ S. D. Kenny, G. Rajagopal, and R. J. Needs, Phys. Rev. A 51, 1898 (1995).

${ }^{34}$ M. Mella, G. Morosi, and D. Bressanini, J. Chem. Phys. 111, 108 (1999).

${ }^{35}$ A. Sarsa, K. E. Schmidt, and W. Magro, J. Chem. Phys. 113, 1366 (2000).

${ }^{36}$ S.-I. Lu, J. Chem. Phys. 114, 3898 (2001).

${ }^{37}$ B. L. Hammond, W. A. Lester, Jr., and P. J. Reynolds, Monte Carlo Methods in Ab Initio Quantum Chemistry, 1st ed. (World Scientific, Singapore, 1994). 